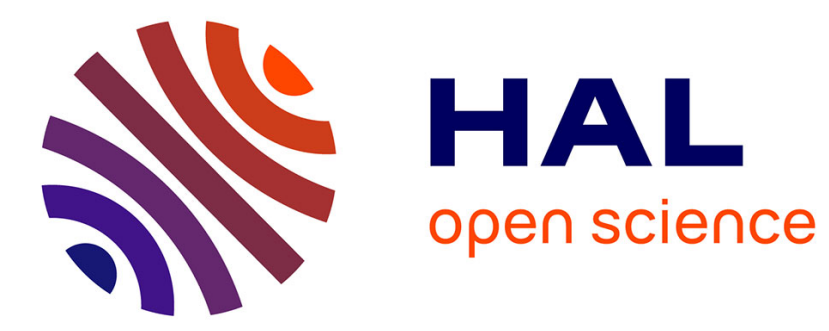

\title{
Improving the use of QFD with Axiomatic Design
}

\author{
António M. Gonçalves-Coelho, António J. F. Mourão, Zulema L. Pereira
}

\section{To cite this version:}

António M. Gonçalves-Coelho, António J. F. Mourão, Zulema L. Pereira. Improving the use of QFD with Axiomatic Design. Concurrent Engineering: Research and Applications, 2005, 13 (3), pp.233-239. 10.1177/1063293X05056787 . hal-00571182

\section{HAL Id: hal-00571182 \\ https://hal.science/hal-00571182}

Submitted on 1 Mar 2011

HAL is a multi-disciplinary open access archive for the deposit and dissemination of scientific research documents, whether they are published or not. The documents may come from teaching and research institutions in France or abroad, or from public or private research centers.
L'archive ouverte pluridisciplinaire HAL, est destinée au dépôt et à la diffusion de documents scientifiques de niveau recherche, publiés ou non, émanant des établissements d'enseignement et de recherche français ou étrangers, des laboratoires publics ou privés. 


\title{
Improving the use of QFD with Axiomatic Design
}

\author{
António M. Gonçalves-Coelho, ${ }^{*}$ António J. F. Mourão and Zulema L. Pereira \\ Faculty of Science and Technology, The New University of Lisbon \\ Campus da Caparica, 2829-516 Caparica, Portugal
}

\begin{abstract}
Concurrent engineering employs a set of functional disciplines to simultaneously perform the tasks that lead to a product satisfying a list of customer needs. Quality function deployment (QFD) is a methodology that has been broadly used to listen to the voice of the customer. This article intends to improve the use of QFD in concurrent engineering by adoption of axiomatic design (AD) principles, namely the independence of functional requirements and the hierarchical decomposition following a zigzag path. The conclusion is that the inclusion of these AD concepts avoids multilevel iterations in the QFD approach, which can significantly reduce the lead time and costs.
\end{abstract}

Key Words: axiomatic design, QFD, concurrent engineering.

\section{Introduction}

The quality of a product depends on several activities, and one of the most important is the design process development. In fact, poor design is the root of roughly $80 \%$ of the perceived product defects and $60 \%$ of the failures occurring in the products' warranty period [1]. Moreover, about $80 \%$ of the manufactured products' costs result from the decisions that were made during the product design process [2].

Before the industrial age, a single human being usually had the necessary knowledge and skills to develop an entire engineering project. However, the increasing intricacy of products, the advent of elaborate technologies, and the escalation of the market's competitiveness changed this scenario. Nowadays, the development of most of the new products calls for multidisciplinary knowledge, and therefore engineering design has evolved into typical team working. This has led to new organizational evolution and concurrent engineering (CE) is one of them.

The CE approach systematically employs a set of functional disciplines to integrate and simultaneously perform all the tasks that yield to a product satisfying a list of customer needs. Its main goals are to shorten the products' time to market, to increase their quality and performance, and to reduce their overall life cycle costs, from conception through disposal.

As a paradigm for the design process, $\mathrm{CE}$ allows for the adoption of some design theories and methodologies,

*Author to whom correspondence should be addressed. E-mail: agc@fct.unl.pt such as quality function deployment (QFD), axiomatic design (AD), design for manufacture and assembly (DFMA), value engineering (VE) and failure mode and effects analysis (FMEA). This article intends to show that axiomatic design can lead to an improved use of QFD to attain a better concurrency.

\section{The Axiomatic Design Theory}

\subsection{Main Principles}

Axiomatic design was developed by N.P. Suh in the late 1970 s to be used as a systematic model for engineering education and practice. Its underlying hypothesis is that there are fundamental principles that govern good design practice $[3,4]$. AD key components are domains, axioms, hierarchies, and zigzagging.

Under the AD point of view, the design outputs pertain to four distinct domains: the customer domain, the conceptual domain, the physical domain and the process domain (see Figure 1).

The design process begins in the customer domain with the identification of the customer needs $(\mathrm{CNs})$, i.e., the features that customers are looking for in the 'design object', be it a product, a process, or any other tangible or intangible system. Mapping between the customer and the conceptual domains is used to find out the functional requirements (FRs) of the design object. Once this is done, another mapping makes the translation of the FRs into design parameters (DPs), which are the set of properties that describe the object in the physical domain. At last, mapping from the physical domain to the process domain leads to the process 


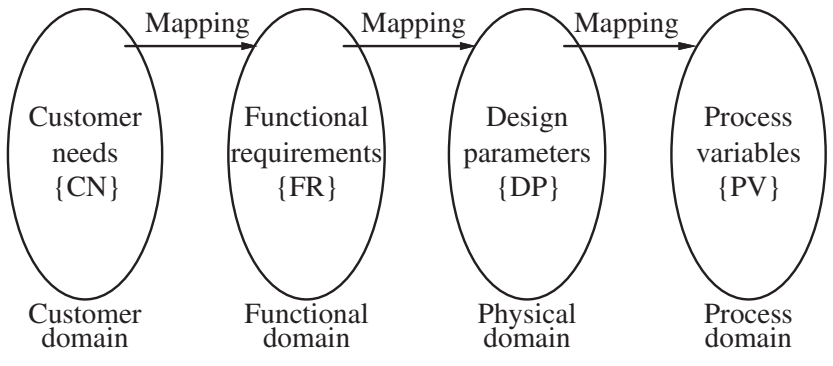

Figure 1. The design process as a mapping through domains.

variables (PVs), which outline how to make the design object.

The so-called input constraints are considered at the outset of the design process. Additional constraints the system constraints - can only be unveiled during the design process development, because they depend on the partial solutions that are adopted later on.

Mapping between any two contiguous domains is represented by a design equation. Mapping from the functional to the physical domains, for example, is given by

$$
\{\mathrm{FR}\}=[\mathrm{A}]\{\mathrm{DP}\}
$$

where $\{\mathrm{FR}\}$ is the functional requirement vector, $\{\mathrm{DP}\}$ is the design parameter vector, and $[\mathbf{A}]$ is the design matrix, whose generic element is

$$
\mathbf{A}_{i j}=\frac{\partial \mathrm{FR}_{i}}{\partial \mathrm{DP}_{j}}, \quad i=1, \ldots, n ; \quad j=1, \ldots, m .
$$

Therefore, $\mathbf{A}_{i j}$ measures the local sensivity of $\mathrm{FR}_{i}$ to the variation of $\mathrm{DP}_{j}$.

Each one of the above-mentioned mapping steps is not unique, and depending on the way they are performed, they may lead either to 'good design' or to 'poor design'. According to $\mathrm{AD}$, good design must comply with the independence and the information axioms.

Independence axiom: Maintain the independence of functional requirements.

Alternative statement: In an acceptable design, mapping between the FRs and the DPs is such that each FR can be satisfied without affecting the other FRs.

As regards the independence axiom, there are three basic design types: uncoupled, decoupled, and coupled. Ideally, the number of DPs in the design equation has to equate the number of FRs, in case of which the equation matrix is squared. The best designs are the uncoupled ones, whose equation matrix is diagonal. If the equation matrix is triangular, then the design is decoupled and is still acceptable. Any other configuration of a squared

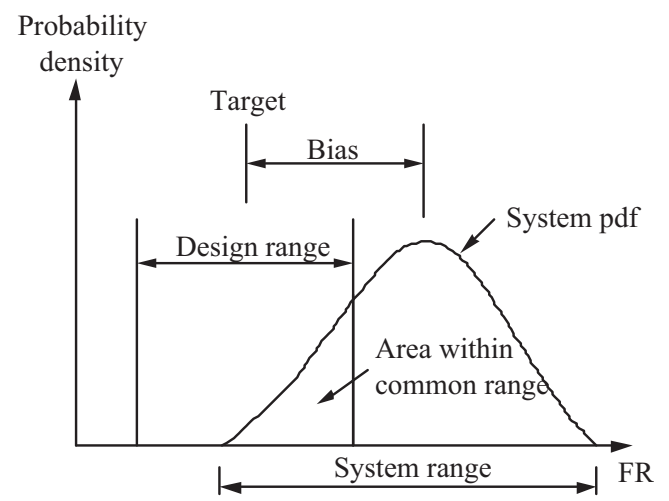

Figure 2. The probability of success for a one-FR, one-DP design (adopted from [4]).

design matrix relates to a coupled design and should be avoided. Moreover, design equations with less DPs than FRs are coupled and should also be avoided. At last, if the number of DPs is larger than that of FRs, then the design is either coupled or redundant. Redundant designs are acceptable, if they are uncoupled or decoupled.

For a given problem, it is most likely that one can find distinct solutions that are acceptable in what to the independence axiom concerns, in case of which the information axiom provides a quantitative means for evaluating their relative merit.

Information axiom: Minimize the information content of the design.

Alternative statement: In a set of designs that satisfy the same FRs and conform to the independence axiom, the best is the one with the minimum information content.

In the simple case of a one-FR, one-DP design, the information content is defined as being the logarithm of the inverse of the probability of achieving the desired value for the FR:

$$
I=\log _{x} \frac{(\text { area of the system range })}{(\text { area of the common range })},
$$

where the area of the system range is the entire area under the FR's probability density function (pdf), and the area of the common range is the fraction of the above-mentioned area that is located inside the design range limits (see Figure 2). Usually, logarithms of base 2 are used, in case of which the information unit is the bit.

The total information content, $I_{\mathrm{t}}$, of an uncoupled design with $n$ FRs, can be computed through

$$
I_{\mathrm{t}}=\sum_{i=1}^{n}-\log _{2} p_{i}=\sum_{i=1}^{n} I_{i}
$$

where $p_{i}$ is the probability of satisfying $\mathrm{FR}_{i}$. Computing the information content of a decoupled design requires 


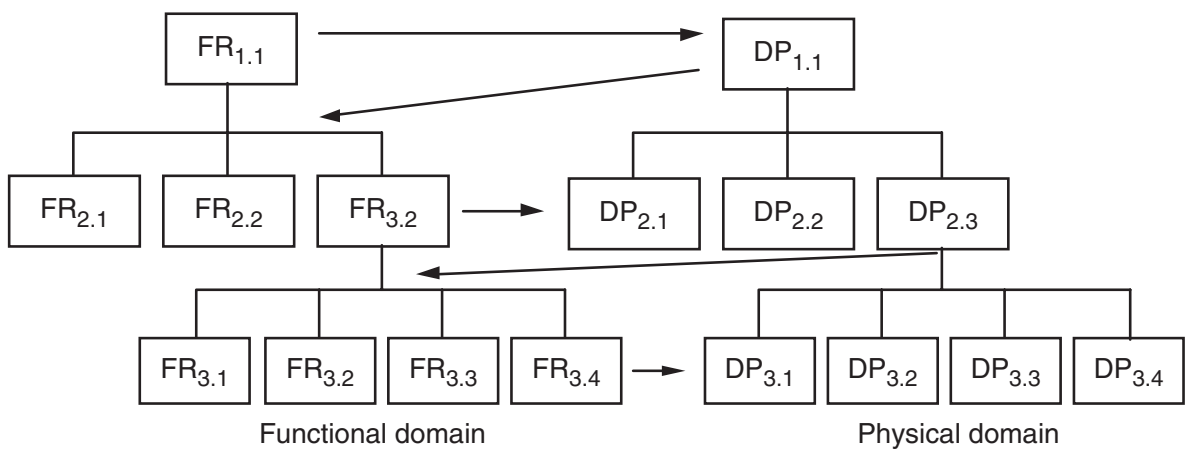

Figure 3. Zigzagging and hierarchical decomposition.

a complex procedure (which is not discussed here) and its amount is never exactly the sum denoted by Equation (4) [5].

\subsection{Hierarchies and Zigzagging}

The design process is mostly synthesis and a unique procedure must be used to achieve the design object decomposition. In fact, there is a difference between analyzing an already existing object and decomposing an object that still does not exist in our mind and is about to be created.

According to $\mathrm{AD}$, the design process must be developed in a top-bottom manner, beginning in the functional domain, at the system level, and continuing through levels of more detail until the point where the design object is defined with sufficient detail. Moreover, to decompose a 'not yet existing object', one has to go back and forth between at least two contiguous design domains, following a zigzag path, as shown in Figure 3. The process is called hierarchical decomposition, and its output comes into view in a fractal-like representation [6]. The result can be depicted by a tree-model in each one of the four design domains.

Zigzag decomposition delineates the design object's hierarchy and turns designing into a controllable process, because the combination of the hierarchy with the design equation unveils the interplay that exists between all the design elements. This may not be especially important for the re-design of existing objects, but is of paramount importance when designing new ones. As will be seen later, the hierarchical decomposition through zigzagging is quite important to achieve a sound CE process.

\section{The QFD Methodology}

QFD is a team-based multifunctional technique meant to provide a systematic way to improve product quality. It was developed in the 1960s, in Japan, and spread to the western countries in the early 1980s [7]. In short, QFD provides a means to catalogue the perceived

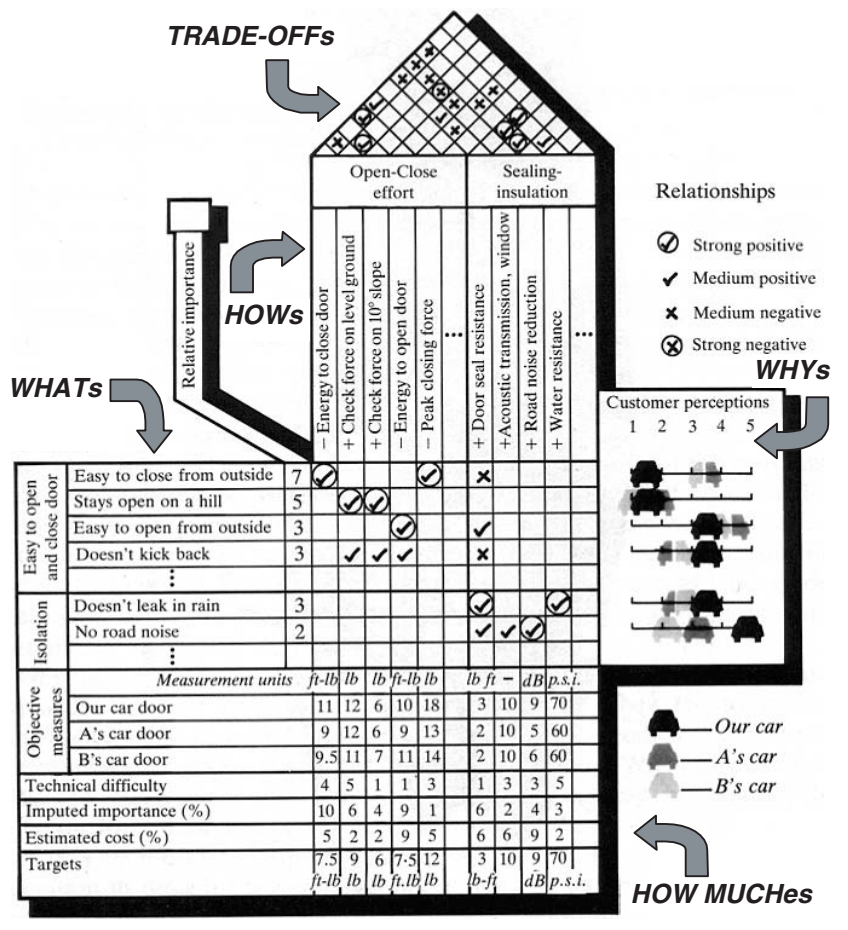

Figure 4. The house of quality (adapted from [8]).

needs of the customer and to translate them into design specifications, all over product planning, product design, process design, and production planning. The key element of QFD is a combined chart that is known as the House of Quality (HOQ), as shown in Figure 4.

The core of HOQ is the relationship matrix, which is used to map the customer attributes or requirements (the 'WHATs') into engineering characteristics (the 'HOWs') that must be adjusted to fulfil the customer needs. The trend and the strength of those relationships are recorded in the relationship matrix with special symbols. The customer attributes are usually bundled according to an affinity criterion.

The 'basement' of the HOQ, which is also called the 'HOW MUCHes', has two fields. The first one, objective measures, summarizes the engineering characteristics as they were measured on the products that are under scrutiny. As for the second, it is meant to 


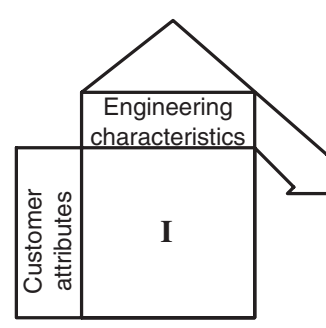

Product planning (the house of quality)

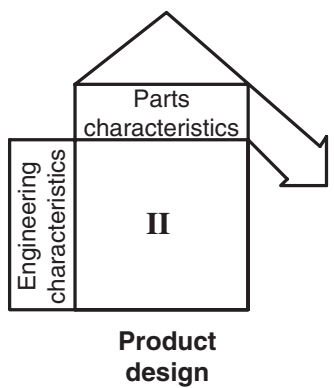

design

Figure 5. The QFD's four-phase design model.

display the results of a competitive benchmarking and to list the target values of the engineering characteristics that are to be achieved.

The 'roof' of the HOQ is the correlation matrix (or 'TRADE-OFFs'), which records all the existing positive and negative interactions between the distinct HOWs. Usually, the positive interactions are considered as redundancy, and the negative ones are regarded as sources of conflict that have to be managed through trade-off procedures.

Finally, the purpose of the 'mezzanine' at the right side of the HOQ, the customer perceptions (or 'WHYs'), is to show how the customers rate the attributes of the distinct competitive products under analysis, including the one that is being developed. These figures help the engineers to perceive the attributes are worth improving.

HOQ does not acknowledge the existence of design constraints, and in an ideal case is applied to all the four sequential phases of the product's development cycle (see Figure 5). Each phase can be deployed in several matrices, depending on the product to be developed.

The four-phase approach provides a comprehensive and appealing chart that allows one to assess the effect of the design changes on the fulfilment of the requirements, and which can be used to implement CE [9]. Let us assume that each QFD phase corresponds to a single matrix and that the HOWs of each matrix become the WHATs of the next one.

\section{Axiomatic Design and QFD}

Considering the QFD phase II (Figure 5), both AD and QFD outline the design process as a mapping between domains, and both the QFD's relationship matrix and the AD's design matrix have the same meaning: they express the sensivity of each WHAT (or FR) relative to each one of the different HOWs (or DPs). However, QFD and AD make use of those matrices in distinct ways.

The criteria generally used in QFD to group the customer needs does not focus on the product's architecture, the knowledge of which is necessary when

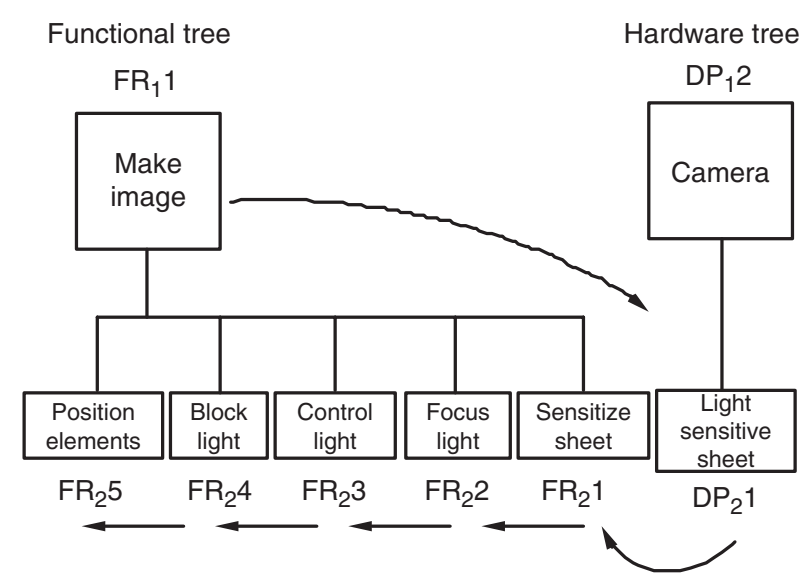

Figure 6. Completing the functions [9].

designing products from the scratch. This deficiency is openly recognized by some of the major QFD experts: in their own words, QFD is not useful for the creation of new products '... because quality deployment stops at the point where quality has been deployed to a level that is extremely difficult to attain. A quality chart created from this type of deployment does not lead to anything new because products of such a quality chart are merely combinations of the available technologies' [10, p. 299].

In an interesting book, Clausing gives the impression of overtaking the above-mentioned flaw by adopting a decomposition technique (see Figure 6) that is roughly inspired by the AD's zigzag approach [9, pp. 233-239].

In our opinion, however, the attempt is not successful because his decomposition procedure is loose in what the zigzagging concerns. In the photo camera example of Figure 6, $\mathrm{FR}_{1} 1$ is used to achieve $\mathrm{DP}_{2} 1$, which belongs to the second level of the functional tree. Then, $\mathrm{DP}_{2} 1$ is used to find all the second level FRs, as shown in Figure 6. He proceeds by coming back to the hardware tree to find out the remaining second level DPs (not shown in the figure). The decomposition ends at this level, with no indication about how to proceed to lower levels, which is frequently required in real design. Additionally, Clausing does not precisely follow AD's zigzagging procedure, and states that the decisionmaking sequence does not need to strictly alternate 


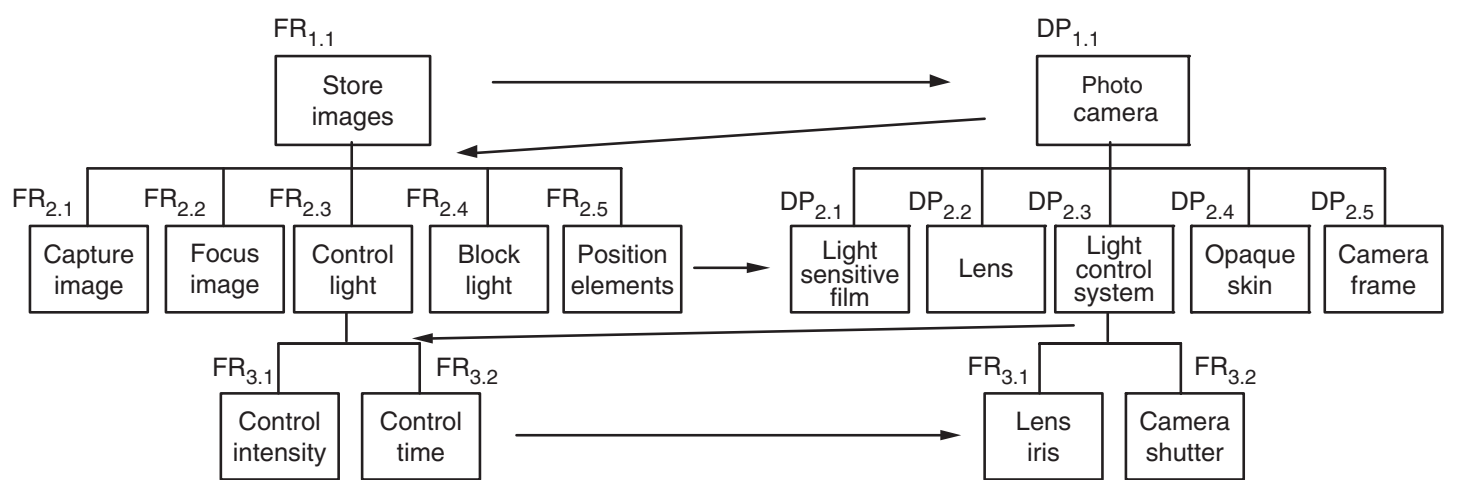

Figure 7. The design of a photo camera.

between functional requirements and design parameters [9, p. 236]. At last, he says that $\mathrm{DP}_{1}$ (the camera) is essentially a tautological name that is not really necessary to the decomposition [9, p. 234].

We have a different point of view: departing from the same top-level functional requirement, 'make image' and not knowing, at the outset, which is the best way to achieve this - our top-level decision would be to choose a type of device that allows making images. It could be a canvas, a mirror, a video camera or, of course, a photo camera. After having chosen the photo camera solution, we would jump to the second level of the functional tree, to find out the main functional requirements that the photo camera should have, as shown in Figure 7. Then, we would use the second level FRs to make more decisions, that is, we would choose the second level physical solutions in the hardware tree. The decomposition would proceed in this way until the appropriate level of detail was achieved.

One can easily realise that each decomposition step of Figure 7 represents a decoupled design. One can also verify that the procedure was achieved following a strict zigzag path, for the decisions that are made at each decomposition level have important consequences in the definition of the lower level FRs [11]. Therefore, the allusion to the photo camera is not tautological for us: should we have chosen a canvas, for example, and at least $\mathrm{FR}_{2.2}$ (focus image), $\mathrm{FR}_{2.3}$ (control light) and $\mathrm{FR}_{2.4}$ (block light) would be meaningless.

Our conclusion is that the camera example of Figure 6 does not depict the decomposition as it should be done in a true design situation, but corresponds to a reverse engineering exercise. Actually, using DPs to generate FRs is a strong challenge to a peacefully accepted design rule, which states that the perception of any desired function (the WHAT, or FR) must precede the search for the corresponding design solution (the HOW, or DP).

We understand that there are many different ways to decompose an object. Pahl and Beitz [1], for instance, do not use the concept of design domains (or trees), and do not zigzag at all. But if we are really designing a new object from the scratch, then we have to strictly alternate

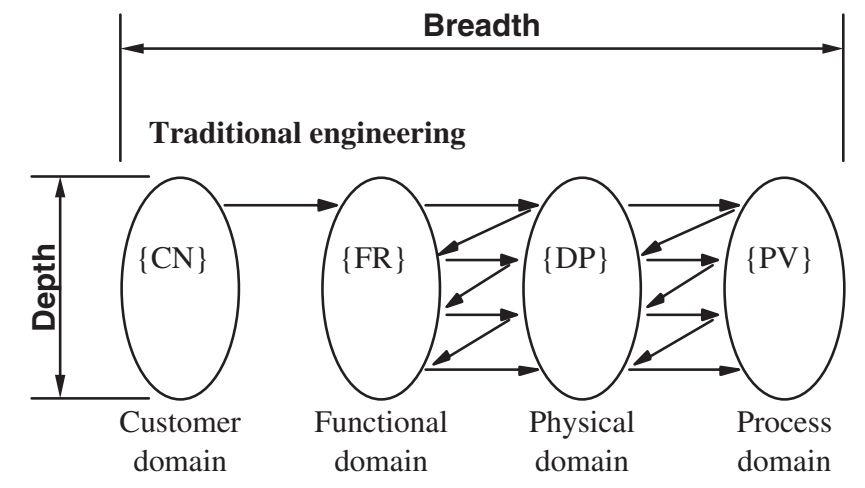

Concurrent engineering

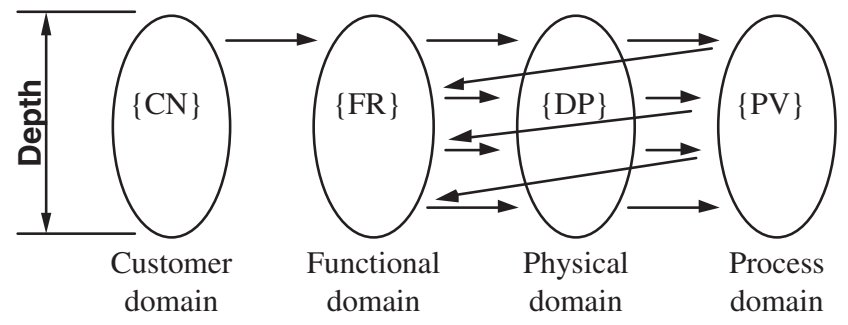

Figure 8. The decomposition process in traditional and in concurrent engineering.

between the design domains. In fact, any coupling that is not solved at a certain decomposition level cannot be decoupled at the lower levels. Moreover, full concurrency depends on nondependency, as we will show here.

Figure 8 depicts the path that is followed during the design decomposition, for both traditional and concurrent engineering processes. One can consider that the design process has two dimensions: breath represents the scope of the design process, and depth the attained level of detail.

Usually, the customer needs are defined in an imprecise language, and hence it is often impossible to hierarchically map the CNs and the FRs by following a zigzagging path. This is a condition where the use of the HOQ can be highly valuable. After acquiring the FRs, there are two possible ways are to perform the decomposition. 
In traditional engineering, one begins zigzagging between the functional and the physical domains until the design object is sufficiently detailed. Next, the zigzagging between the physical and process domain is performed. It may happen, however, that one cannot find out any acceptable solution for the entire depth of the DP to PV decomposition. In this case, it is most likely that a substantial part of the FR to DP mapping has to be repeated in both the breath and the depth direction. This may represent a significant loss of time and of other resources.

In concurrent engineering, on the other hand, the zigzag path encompasses the entire design breadth (with the possible exception of the $\mathrm{CN}$ to FR mapping) and this is why nondependency is important. In this case, some iterations at any given decomposition level may be necessary, but it is guaranteed that there is no need to reiterate the higher decomposition levels. This means that the probability of success in the use of the HOQ is higher if the grouping of the WHATs and the HOWs is carried out under the AD principles, by grouping them according to the decomposition level to where they belong.

\section{Further Remarks}

Current QFD makes no discernible distinction between requirements and constraints. This is quite perceptible in the example of the car's door in Figure 4, wherein can find requirements such as 'doesn't quick back' and 'no road noise'. In our opinion, nonassertive statements should not be assigned as requirements, but rather as constraints. This would allow the diminishing of the number of requirements. Notice that according to the $\mathrm{AD}$ approach, the lesser the number of DPs, the easier it is to achieve uncoupled or decoupled design solutions.

On the other hand, common QFD lacks a broad criterion to assess the performance of alternative design solutions. In fact, QFD uses benchmarking, which is satisfactory when one is trying to improve an existing design by comparing it with alternative solutions. However, when a new design must be developed from scratch, there are no prototypes to benchmark. In this case, the full use of the HOQ is possible only in advanced stages of product development, when prototypes become available.

One may wonder if the disregard to the product's architecture and the lack of comprehensive assessment criteria are the true reasons why so many designers use QFD just to perform product planning [12, p. 68, 309], thus failing to achieve concurrent engineering as they were supposed to. In fact, current QFD challenges the designers at improving the quality of the design objects just by enhancing the quality of its components, not stimulating to the search for alternative design solutions with a better architecture.

\section{Conclusions}

Quality function deployment is a widespread design methodology, whose most impressive feature is the House of Quality, an ingenious and comprehensive layout to document of the whole design process.

In its current form, QFD is a good tool for improving existing designs, but is not the most suitable instrument for the concurrent development of new products, because: (1) it does not capture the design object architecture, which is crucial for the design of new objects and for the in depth redesign of already existing ones; (2) it does not make use of consistent criteria to empower the engineers to make good decisions, especially in the early steps of the design decomposition process; (3) it is not intrinsically concurrent and does not encourage the designers to search and compare alternative design solutions.

In the present article, it is clear that AD's design matrix and QFD's relationship matrix represent the same reality, differing only in their format.

Using simple examples, it was also shown that AD provides: (1) a systematic procedure to decompose the design object that captures its architecture; (2) a set of unchanging criteria that is essential to promptly assess the engineering design decisions at any point of the design process, with a strong focus on the FR nondependency; (3) an intrinsically concurrent working background that promotes the fast generation of new design solutions.

Therefore, the main advantage of including the principles of $\mathrm{AD}$ in the $\mathrm{QFD}$ implementation is to avoid multilevel (in depth) iterations. This fact can significantly reduce the lead time and, consequently, decrease costs and improve the competence of the organization.

\section{Acknowledgments}

The authors would like to thank the Faculty of Science and Technology of The New University of Lisbon, and the Portuguese Foundation for Science and Technology for their valuable support to our research work. The authors are also very grateful to the reviewers for their useful suggestions.

\section{References}

1. Pahl, G. and Beitz, W. (1999). Engineering Design: A Systematic Approach, 2nd edn, London: Springer-Verlag.

2. Bakerjian, R. (ed.) (1992). Tool and Manufacturing Engineers Handbook, Design for Manufacturability, Vol. VI, Dearborn, Michigan: Society of Manufacturing Engineers (SME).

3. Suh, N.P. (1990). The Principles of Design, New York: Oxford University Press. 
4. Suh, N.P. (2001). Axiomatic Design: Advances and Applications, New York: Oxford University Press.

5. Frey, D.D., Jahanjir, E. and Engelhart, F. (2000). Computing the Information Content of Decoupled Designs, In: 1st International Conference on Axiomatic Design, Cambridge, Mass, June 21-23, pp. 151-161.

6. Hintersteiner, J.D. (1999). A Fractal Representation for Systems, In: The 1999 International CIRP Seminar, Enschede, Netherlands, March 24-26.

7. Akao, Y. (1997). QFD: Past, Present, and Future, In: International Symposium on $Q F D^{\prime}$ 97, Linköping, Sweden, October.

8. Hauser, J.R. and Clausing, D. (1988). The House of Quality, Harvard Business Review, 66(3): 63-73.

9. Clausing, D. (1994). Total Quality Development: A Stepby-Step Guide to World-Class Concurrent Engineering, New York: ASME Press.

10. Mizuno, S. and Akao, Y. (1994). QFD: The CustomerDriven Approach to Quality Planning and Deployment, Tokyo: Asian Productivity Organization.

11. Lindholm, D., Tate, D. and Harutunian, H.(December 1999). Consequences of Design Decisions in Axiomatic Design, Trans. of the Society for Design and Process Science, 3(4): 1-12.

12. Cohen, L. (1995). Quality Function Deployment: How to Make QFD Work for You, Reading, Mass: Addison-Wesley Pub. Co.

\section{António M. Gonçalves-Coelho}

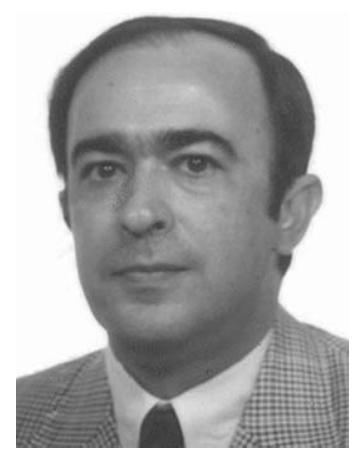

António M. GonçalvesCoelho has a $\mathrm{PhD}$ degree in Mechanical Engineering. Currently, he is an Associate Professor of Machine Design, coordinates the Machine Design Group of the Mechanical and Industrial Engineering Department at the Faculty of Science and Technology, The New University of
Lisbon, and is a member of UNIDEMI (Research and Development Unit on Mechanical and Industrial Engineering).

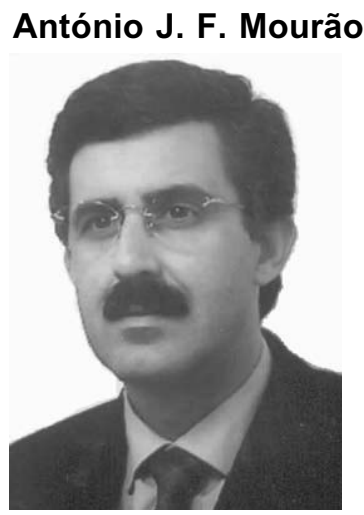

António J. F. Mourão has an $\mathrm{MSc}$ and a $\mathrm{PhD}$ degree in Mechanical Engineering. Currently, he is an Assistant Professor, coordinates the Industrial Technology Group of the Mechanical and Industrial Engineering Department at the Faculty of Science and Technology, The New University of Lisbon, and is a member of UNIDEMI (Research and Development Unit on Mechanical and Industrial Engineering).

\section{Zulema L. Pereira}

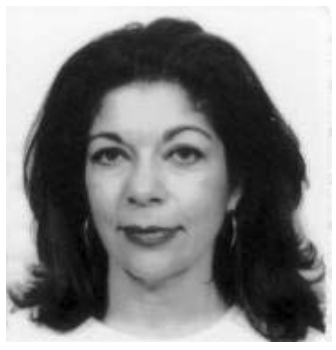

Zulema L. Pereira has an MSc degree in Operational Research and Systems Engineering and a $\mathrm{PhD}$ degree in Industrial Engineering. Currently, she is an Associate Professor of Quality Engineering, coordinates the Quality

Engineering Group of the Mechanical and Industrial Engineering Department at the Faculty of Science and Technology, The New University of Lisbon, and is a member of IET (Research Centre on Enterprise and Research Innovation). 